\title{
A Study on the Number of Brushing Strokes in Toothbrushing Education
}

\author{
Yong-Keum Choi ${ }^{*}$, Jin-Sun Choi ${ }^{1, *}$, Keun-Ok Lim, Deok-Young Park ${ }^{1}$, and Yoonhee Kim ${ }^{2, \dagger, \neq}$ \\ Department of Dental Hygiene, College of Health Science and Genome-based BiolT Convergence Institute, \\ Sun Moon University, Asan 31460, \\ ${ }^{1}$ Department of Preventive and Public Health Dentistry, College of Dentistry and Research Institute of Oral Science, \\ Gangneung-Wonju National University, Gangneung 25457, Korea, \\ ${ }^{2}$ Institute of Tropical Medicine, Nagasaki University, Nagasaki 852-8523, Japan
}

\section{칫솔질 교육 시 칫솔질 행정횟수에 관한 연구}

\author{
최용금* $\cdot$ 최진선 ${ }^{1, *} \cdot$ 임근옥 $\cdot$ 박덕영 ${ }^{1} \cdot$ 김윤희 $^{2, \dagger, キ}$ \\ 선문대학교 건강보건대학 치위생학과 및 유전체 기반 바이외 융합 연구소, '강릉원주대학교 치과대학 예방치학교실 및 구강과학연구소, \\ ${ }^{2}$ 나가사키대학교 열대의학연구소
}

\begin{abstract}
The purpose of this study was to focus on the number of brushing strokes among the performance factors and identify if the 10 times stroke of the rolling method is rational. Moreover, we evaluated the changes in oral health knowledge, perception, and behavior after our rolling method instruction. The 10-stroke method of toothbrushing has been regarded as an effective method of removal of dental plaque, although there is little evidence to support this claim. We allocated 40 healthy subjects to two intervention groups. During five visits, we measured a score for dental plaque removal and instructed the subjects on a toothbrushing technique with 5 or 10 strokes per section. At the initial and final visits, subjects completed a questionnaire on one designed specifically for this study about oral health knowledge, perceptions, and behaviors. Repeated measures analysis of variance was used to compare the dental plaque removal score between the groups, and the changes in scores within each group over time. We also compared changes in mean scores in oral health knowledge, perceptions and behaviors before and after toothbrushing instruction. We found that the score for dental plaque removal increased with each additional toothbrushing instruction in both groups $(p<0.001)$. However, we found no differences in the dental plaque removal scores between the 5 -stroke and 10-stroke groups $(p=0.399)$. The levels of oral health knowledge, perceptions and behaviors increased after the toothbrushing instructions in both groups. Our findings suggest that there is no advantage in emphasizing the 10-stroke method of toothbrushing in an oral health education program.
\end{abstract}

Key Words: Dental plaque, Health education, Oral health, Toothbrushing

\section{Introduction}

Dental caries and periodontitis are major chronic oral diseases and leading causes of tooth loss ${ }^{1}$. Dental plaque plays an important role as an environmental factor in the oral cavity to exacerbate dental diseases by harboring pathogenic bacteria that cause inflammation ${ }^{2}$. However, these dental diseases are preventable by removing the dental plaque mechanically (e.g., toothbrushing, flossing, interdental brushing, and oral prophylaxis) and by Tel: +81-(0)3-5841-3582, Fax: +81-(0)3-5841-3582, E-mail: yoonheekim @ m.u-tokyo.ac.jp, ORCID: https://orcid.org/0000-0003-2517-1087 
weakening the pathogenic activity by chemical means (e.g., mouth rinses and drug treatment ${ }^{3,4)}$. Toothbrushing is the most commonly used method of mechanical self-care and is regarded as effective when used with toothpaste ${ }^{5)}$. Toothbrushing applies the toothpaste components to the teeth and gingiva and stimulates the gingiva, which helps to prevent dental diseases ${ }^{6}$.

Toothbrushing instruction is a key part of any oral health promotion program that aims to improve people's knowledge, attitudes, and behaviors in oral health. It enables people to develop their self-care skills to control dental hygiene ${ }^{7)}$. During the instruction, although it is important to provide enough time and repetition to learn the skills, oral health instructors can achieve the educational goals in a limited time with a validated instruction manual based on the rationale for toothbrushing ${ }^{8)}$.

There are several techniques for toothbrushing. Dental professionals in South Korea have taught the rolling method as the primary brushing technique, except in special cases, such as people with orthodontic appliances. The rolling method involves placing the bristles against the gingiva parallel with the vertical plane of the tooth, and sweeping the bristles up to the occlusal surface using wrist power ${ }^{9)}$. The overall sequence of this method consists of grasping a toothbrush, making the rolling motion described above, repeating the motion a certain number of times, covering all surfaces of the teeth and the tongue ${ }^{10)}$.

However, there is a lack of scientific evidence of the rolling method particularly in its performance factors. For example, some research questions could be potentially addressed: if the palm grasp method is the best way in brushing, if the 10 times stroke of brushing is meaningful, whether the stroke motion rolling a toothbrush is more efficient than another type of motions, if each section of teeth should be evenly brushed, and what would contribute the most to remove the dental plaque among the performance factors. In general, previous studies have shown the total effect of the rolling method before and after their education program ${ }^{11,12)}$. Although some studies compared the removal of dental plaque between the rolling and Bass methods, the performance factors were not considered ${ }^{9,10}$ )

Dental professionals in South Korea have recommended brushing with at least 10 strokes for every section corresponding to the length of the toothbrush head when using the rolling method ${ }^{13)}$. However, many people in South Korea tend not to brush thoroughly than this recommendation $^{14)}$. Also, there is no such a recommendation in the Western countries in their toothbrushing instruction guidelines ${ }^{15,16)}$. They have rather recommended the Bass method ${ }^{17-19)}$

Therefore, it is merited to provide more evidence of the rolling method to ascertain what is an appropriate way to recommend as a primary brushing method, and if so, how to use it properly. In this study, we aim to focus on the number of brushing strokes among the performance factors and examine if the 10 times stroke of the rolling method is rational. Additionally, we examined the changes in oral health knowledge, perception, and behavior after our rolling method instruction.

\section{Materials and Methods}

\section{Study subjects}

We recruited 40 healthy non-smoking students aged in their 20s, with no orthodontic appliances, from Sun Moon University, South Korea. We allocated the participants to two intervention groups with different toothbrushing regimes. To control potential confounders, we matched the participants in one group with the other by sex, the experience of toothbrushing instruction, type of the brushing method in the instruction, level of interest in toothbrushing, and oral health knowledge obtained at the first interview (baseline visit) in our study. There was no difference in the matching variables between the two groups ( $\mathrm{p}<0.05)$. We also measured the number of strokes used by the subjects when toothbrushing and obtained an average value of 4.5 strokes. We thus defined the average stroke number as five, and the recommended stroke number as 10 . In statistical analyses, eight subjects were excluded due to their low attendance. Of the 32 subjects remained, the two groups finally included 17 and 15 subjects respectively. The Public Institutional Review Board from the Sun Moon University approved our study protocol and consent forms (PIRB12-056-1). We obtained informed consent from all participants. 


\section{Study design}

This study is an experimental study. Experimental studies compare the results of study participants assigned to an intervention group and a control group. Therefore, in this experiment, the group of 10 strokes that are generally educated about the number of brush strokes and the group of 5 brush strokes that people are performing on the average were set as the control group.

Fig. 1 shows the flowchart in our study. At the baseline visit, we interviewed all subjects to collect demographic characteristics and data about their knowledge, perceptions, and behaviors in oral health. They also underwent an oral examination to assess their dental plaque levels. The interview questionnaire for oral health consisted of nine items (three items each for knowledge, perceptions, and behaviors) as listed in Table 1, adapted from a previous study. Some of these items were used as the matching variables described above. We used a five-point scale for each question (i.e., 'entirely agree,' 'agree,' 'neutral,' 'disagree,' and 'entirely disagree') corresponding to the

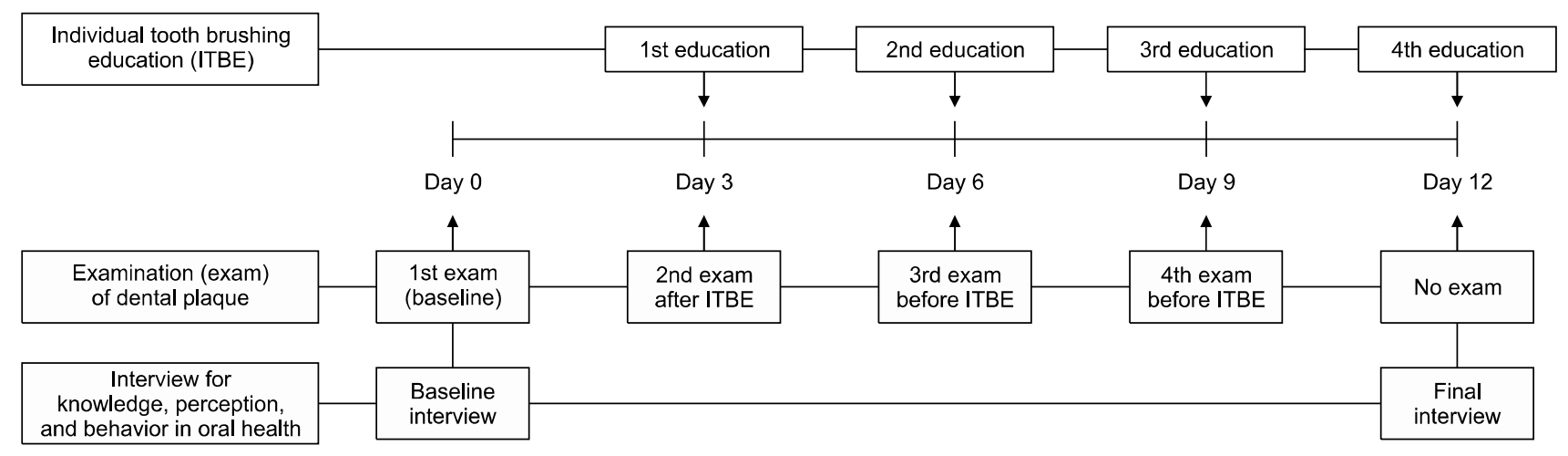

Fig. 1. Flow chart of this study.

Table 1. Modified O'Leary Index and Oral Health Knowledge, Perceptions, and Behaviors between the Groups at the Baseline Visit

\begin{tabular}{|c|c|c|c|}
\hline Category & 5-Stroke $(n=17)$ & 10 -Stroke $(n=15)$ & p-value \\
\hline Modified O’Leary index & $51.0 \pm 18.0$ & $51.4 \pm 18.1$ & 0.951 \\
\hline No experience in tooth brushing education & 94.1 & 100.0 & 0.340 \\
\hline \multicolumn{4}{|l|}{ Oral health knowledge } \\
\hline Total score of knowledge & $10.5 \pm 1.6$ & $10.8 \pm 1.8$ & 0.657 \\
\hline Fluoride prevents dental caries & 41.2 & 60.0 & 0.288 \\
\hline Severe periodontitis can destroy teeth with surrounding bones & 76.5 & 60.0 & 0.316 \\
\hline An adult has permanent teeth of $28 \sim 32$ & 47.1 & 66.7 & 0.265 \\
\hline \multicolumn{4}{|l|}{ Oral health perception } \\
\hline Total score of perception & $11.0 \pm 1.4$ & $10.6 \pm 1.2$ & 0.486 \\
\hline Oral diseases are preventable under control & 82.4 & 100.0 & 0.229 \\
\hline Tooth brushing in a proper way is helpful to prevent oral diseases & 94.1 & 93.3 & $<1.000$ \\
\hline I know how to control my dental hygiene properly & 17.6 & 0.0 & 0.229 \\
\hline \multicolumn{4}{|l|}{ Oral health behavior $^{\mathrm{a}}$} \\
\hline Total score of behavior & $7.1 \pm 1.9$ & $7.4 \pm 1.5$ & 0.661 \\
\hline I brush my teeth before going to bed & 76.5 & 93.3 & 0.338 \\
\hline I go to a dental clinic for a regular check & 0.0 & 6.7 & 0.469 \\
\hline I use auxiliary products in dental hygiene such as floss and an interdental brush & 11.8 & 6.7 & $<1.000$ \\
\hline
\end{tabular}

Values are presented as mean \pm standard deviation or $\%$.

p-values were calculated by the two-samples independent t-test for the modified O'Leary index and the total scores of oral health knowledge, perceptions, and behaviors, and by the chi-square test with Fisher's exact test (the cells less than 5) for each question of oral health knowledge, perceptions, and behaviors.

a Original five-point scales were dichotomized: 'entirely agree' and 'agree' as 'yes'; 'neutral', 'disagree', and 'entirely disagree' as 'no'. 
score from 5 to 1 . Higher scores indicated better knowledge of oral health, and positive perceptions and behaviors for the prevention of oral diseases, and willingness to undertake preventive practices. Across three visits with an interval of 3 days, we carried out one-on-one toothbrushing instruction and measured dental plaque levels at every visit. On the days between visits, we sent the subjects a text message to encourage them to keep using the toothbrushing method as instructed. At the final visit, we interviewed the subjects again to measure changes in their oral health knowledge, perceptions and behaviors, and delivered the final toothbrushing instruction.

The dental plaque examination was based on the modified O'Leary index. For each subject, we applied a disclosing agent to all teeth and counted the number of colored surfaces, excluding the occlusal surface. We then calculated the percentage of surfaces disclosed over the total number of surfaces and inverted the percentage to give the subjects more intuitive information (so that a higher score indicated better dental plaque removal). This procedure of examination was carried out in the same clinical laboratory setting, and all subjects used identical toothbrushes and toothpaste as distributed to them.

Based on the instruction of the dental plaque coloring examination procedure, the following procedure was performed $^{20)}$. The examiner spent 8 minutes total time measuring the dental plaque coloring for 2 minutes and the tooth examination after 6 minutes.

Before recruiting the subjects, we trained four senior students majoring in dental hygiene to perform the tooth- brushing instruction or the dental plaque examination. Two of the students undertook five sessions to study the guidelines and materials for the toothbrushing instruction. The other two examiners demonstrated their technique of dental plaque examination for six adults three times, and obtained reasonably high levels of inter-examiner reliability $(\kappa=0.7)$.

\section{Statistical analysis}

We used an independent two-sample t-test and a chisquare test to match and identify any differences in oral health knowledge, perceptions and behaviors, and in the modified O'Leary index between the five-stroke group and the 10-stroke group at the baseline visit. Also, we performed a repeated measure ANOVA to examine whether the modified O'Leary index changed over time with each successive toothbrushing instruction session. We calculated a total score of the subjects' oral health knowledge, perceptions, and behaviors for each, and used a paired samples t-test to test if the scores changed from the baseline visit to after the last session of toothbrushing instruction in the 5-stroke and 10-stroke groups. All analyses were performed with IBM SPSS statistics ver. 18.0 (IBM Co., Armonk, NY, USA). The significance level was the alpha of 0.05 .

\section{Results}

Table 1 shows summary statistics of the modified O'Leary index and the oral health knowledge, perceptions,

Table 2. Subtotal Scores in Oral Health Knowledge, Perceptions, and Behaviors in 5-Stroke and 10-Stroke Groups before and after the Repeated One-on-One Toothbrushing Instruction

\begin{tabular}{llrrc}
\hline Category & Group & Before & After & p-value \\
\hline Knowledge $^{\mathrm{a}}$ & 5-Stroke & $10.5 \pm 1.6$ & $11.7 \pm 1.2$ & 0.037 \\
& 10-Stroke & $10.8 \pm 1.8$ & $12.1 \pm 1.0$ & 0.022 \\
Perceptions $^{\mathrm{a}}$ & 5-Stroke & $11.0 \pm 1.4$ & $12.0 \pm 2.0$ & 0.040 \\
& 10-Stroke & $10.6 \pm 1.2$ & $12.1 \pm 0.9$ & 0.001 \\
Behaviors $^{\mathrm{a}}$ & 5-Stroke & $7.1 \pm 1.9$ & $8.4 \pm 1.9$ & 0.006 \\
& 10-Stroke & $7.4 \pm 1.5$ & $8.3 \pm 1.9$ & 0.034 \\
\hline
\end{tabular}

Values are presented as mean \pm standard deviation.

p-values were calculated by the paired samples t-test comparing the scores before and after the instruction.

${ }^{a}$ A five-point scale for each question (i.e., 'entirely agree', 'agree', 'neutral', 'disagree', and 'entirely disagree') corresponding to the score from 5 to 1 was summed in each category for oral health knowledge, perceptions, and behaviors, which consists of three questions respectively. 
and behaviors at the baseline visit. There was no difference between the two groups ( $p>0.05$ ). Males were dominant in both groups $(76.5 \%$ and $73.3 \%$ in the 5 -stroke and 10-stroke groups, respectively). Most subjects responded that they had no experience of learning how to brush their teeth $(94.1 \%$ and $100.0 \%$ in the 5 -stroke and 10-stroke groups, respectively). Their knowledge about oral health was moderate; approximately half of the subjects responded with correct answers. Most subjects believed that oral diseases are preventable $(82.4 \%$ and $100.0 \%$ in the 5-stroke and 10-stroke groups, respectively), but they were less confident about the methods they had been using to prevent oral diseases $(17.6 \%$ and $0.0 \%$ in the 5-stroke and 10-stroke groups, respectively). Additionally, some subjects used auxiliary items such as interdental brushes $(11.8 \%$ and $6.7 \%$ in the 5 -stroke and 10 -stroke groups, respectively) and went to the dental clinic for regular checks $(0.0 \%$ and $6.7 \%$ in the 5 -stroke

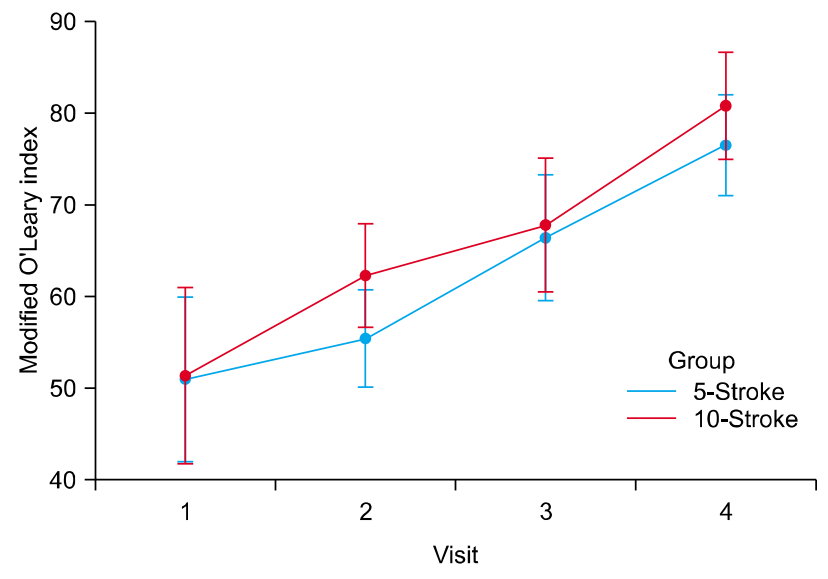

Fig. 2. Estimated marginal means and $95 \%$ confidence intervals of modified O'Leary index over time (visit) in two groups. and 10-stroke groups, respectively). The scores in oral health knowledge, perceptions, and behaviors significantly improved after all participants had taken the repeated one-on-one toothbrushing instruction four times during our study (Table 2).

Fig. 2. shows the changes in the modified O'Leary index over time between the two groups. We found that the mean score significantly increased in both groups as the participants completed further toothbrushing instruction sessions (51.0 to 76.5 in the 5-stroke group and 51.4 to 80.8 in the 10 -stroke group) $(\mathrm{p}<0.001$; Table 3$)$. Also, all of the pair comparisons were significantly different $(p<0.05$ after adjustment for multiple comparisons by Bonferroni correction). However, there was no strong evidence that the mean scores were different between the 5-stroke group and the 10-stroke group ( $\mathrm{p}=0.399$; Table 3$)$.

\section{Discussion}

There are several contributing factors involved in the removal of dental plaque by toothbrushing, such as the strength of the bristles, brushing pressure, the number of strokes, and the type of stroke ${ }^{10)}$. In this study, we aimed to examine whether the removal of dental plaque could be influenced by the number of toothbrushing strokes per section. We found no evidence that the level of dental plaque removal was different between the 5-stroke and 10-stroke groups, although there was a significant improvement in dental plaque removal with each successive toothbrushing instruction session.

However, the results do not suggest that the 5-stroke is the best choice when using the rolling method. It suggests

Table 3. Modified O'Leary Index between Groups of 5-Stroke and 10-Stroke and within the Groups as the Toothbrushing Instruction Sessions Increase (Over Time)

\begin{tabular}{lcccc}
\hline \multirow{2}{*}{ Visit } & \multicolumn{2}{c}{ Stroke frequency of toothbrushing } & & \multicolumn{2}{c}{ p-value } \\
\cline { 2 - 3 } \cline { 5 - 5 } & 5 5-Stroke & W & Within groups & Between groups \\
\hline Visit 1 (baseline) & $51.0 \pm 18.0$ & $51.4 \pm 18.1$ & & \\
Visit 2 & $55.5 \pm 10.4$ & $62.3 \pm 11.0$ & $<0.001$ & 0.399 \\
Visit 3 & $66.4 \pm 15.9$ & $67.8 \pm 10.7$ & & \\
Visit 4 & $76.5 \pm 13.6$ & $80.8 \pm 7.3$ & & \\
Visit 1 $\sim 4$ & $62.3 \pm 17.5$ & $65.6 \pm 16.1$ & & \\
\hline
\end{tabular}

Values are presented as mean \pm standard deviation.

p-values were calculated by repeated measures ANOVA. 
that it is unnecessary to encourage patients to use a greater number of toothbrushing strokes (e.g., 10 strokes per section, as commonly recommended in oral health education in South Korea). A smaller number of strokes (e.g., five strokes per section, as was the average in this study) may be enough to effectively remove dental plaque, together with repeated toothbrushing instruction. Kim et al. $^{21)}$, also suggested that it is needed to re-consider recommending the rolling method only to the public because the number of patients with periodontal disease is increasing. It implies that it is no longer desirable to apply the same toothbrushing method and the number of strokes to everyone. Scientific evidence of the toothbrushing performance factors should be provided to refine the recommendation.

To date, there have been few studies reporting the number of toothbrushing strokes, despite the number of studies comparing toothbrushing methods. Chang and $\mathrm{Kim}^{22)}$ reported that the average number of toothbrushing strokes in the general population in South Korea was fewer than 10 per section. Shin et al. ${ }^{23)}$ suggested that it is necessary to re-evaluate methods of toothbrushing instruction to give more weight to the sequence of toothbrushing and the frequency of toothbrushing how many times a day. They also suggested that five toothbrushing strokes per section may be enough to effectively remove dental plaque.

Another notable finding in this study was that the removal of dental plaque improved following repeated individual toothbrushing instruction regardless of the stroke group. Additionally, we found that the repeated instruction led to significant improvement in the oral health knowledge, perceptions, and behaviors within each group. These findings are consistent with a previous study that reported improved removal of dental plaque from subjects provided with individual-level repeated education compared with those provided with group-level education $^{24)}$. Other previous studies suggested individual repeated toothbrushing instruction rather than one-time group instruction, because the educational effect reverted to the pre-education level after a certain period ${ }^{25,26)}$. Eom et al. ${ }^{27)}$ also reported that repeated individual toothbrushing instruction changed oral health knowledge, perceptions and behaviors, and significantly improved the dental plaque index in their study. Repeated individual toothbrushing instruction was also effective for children in a previous study $^{28)}$. They reported that the method of instruction improved children's knowledge about dental caries motivated their interest of self-care for oral health promotion, and increased their daily toothbrushing frequency. Therefore, we suggest that toothbrushing instruction should be presented individually and repeatedly to habituate learners to brushing their teeth correctly.

There are several limitations in this study. First, we did not consider other factors such as toothbrushing pressure or the extent of toothbrushing skill that might potentially confound the relationship between the number of toothbrushing strokes and the dental plaque score. Second, the dental plaque scores we reported may have been overestimated because we measured the dental plaque score in a clinical laboratory setting, rather than in daily life. Although we provided the same environment to all subjects when measuring dental plaque, it is possible that some subjects may have over performed because of nervousness in the clinical setting. Last, our findings may not be generalizable to other countries using different toothbrushing methods, because we conducted this study based on the rolling method recommended in South Korea.

Our findings suggest that the toothbrushing instruction currently used in oral health education programs should be re-evaluated. Consideration should be given to amending the recommended number of toothbrushing strokes per section. Further studies are needed to clarify the sequence of toothbrushing and how this affects the effective removal of dental plaque. This may lead to a better understanding of the priority of the sequence, and ways in which instructors can present toothbrushing techniques in a limited time.

Furthermore, there is no agreement on effective toothbrushing techniques and the recommended method remains widely diverse depending on a country or a source such as dental associations, dental textbooks, and companies for dental products ${ }^{29,30)}$. More studies are needed to provide evidence of toothbrushing methods to be potentially standardized and recommended to the public.

The findings of our study suggest that the use of a 10 -stroke per section technique with the rolling method is 
not the most important factor in achieving good oral hygiene. It may be more important to determine which teeth are less likely to be cleaned by identifying where dental plaque remains after cleaning, and making an effort to brush all surfaces without exception. We suggest that education about toothbrushing techniques should be reevaluated to provide dental professionals and oral health educators with better guidelines for instructing patients and the public in toothbrushing methods according to the currently available evidence.

\section{Acknowledgements}

This study was supported by research fund from the National Research Foundation of Korea (NRF-2017R1C 1B5017185).

\section{References}

1. Müller A, Hussein K: Meta-analysis of teeth from European populations before and after the 18th century reveals a shift towards increased prevalence of caries and tooth loss. Arch Oral Biol 73: 7-15, 2017.

https://doi.org/10.1016/j.archoralbio.2016.08.018

2. Kim S, Park S, Lin M: Permanent tooth loss and sugarsweetened beverage intake in U.S. young adults. J Public Health Dent 77: 148-154, 2017. https://doi.org/10.1111/jphd.12192

3. Bowen DM: Toothbrushing. In: Darby ML, Walsh M, eds. Dental hygiene: theory and practice. 4th ed. Elsevier, St. Louis, pp.397-405, 2015.

4. Bowen DM: Mechanical oral biofilm control: interdental and supplemental self-care devices. In: Darby ML, Walsh M, eds. Dental hygiene: theory and practice. 4th ed. Elsevier, St. Louis, pp.406-419, 2015.

5. Ray TS: Oral infection control: toothbrushes and toothbrushing. In: Wilkins EM, ed. Clinical practice of the dental hygienist. 9th ed. Lippincott Williams \& Wilkins, Baltimore, pp.402-420, 2005.

6. Nathe $\mathrm{CN}$ : Toothbrushes and toothbrushing methods. In: Harris NO, Garcia-Godoy F, Nathe CN, eds. Primary preventive dentistry. 8th ed. Pearson, Essex, pp.145-160, 2014.

7. Naidu R, Nunn J, Irwin JD: The effect of motivational interviewing on oral healthcare knowledge, attitudes and behaviour of parents and caregivers of preschool children: an exploratory cluster randomised controlled study. BMC Oral Health 15: 101, 2015. https://doi.org/10.1186/s12903-015-0068-9

8. Rossi GN, Sorazabal AL, Salgado PA, Squassi AF, Klemonskis GL: Toothbrushing procedure in schoolchildren with no previous formal instruction: variables associated to dental biofilm removal. Acta Odontol Latinoam 29: 82-89, 2016.

9. Choi YK, Park DY: Comparison of rolling method with Bass method toothbrushing practices for efficacy in plaque removal and degree of easiness. J Korean Acad Oral Health 32: 329-338, 2008.

10. Choi YK, Park DY, Jeong DB: Relationship among adequacy, awareness of the difficulty in toothbrushing and plaque score. J Korean Acad Oral Health 33: 192-200, 2009.

11. Shin SJ, Jang JY, Kim CH: The oral health behaviors for children and teachers by teacher-supervised toothbrushing in community child center. J Dent Hyg Sci 12: 572-582, 2012.

12. Yoon SG, Han SJ: Factors influencing the toothbrushing behavior of elementary school students adapted by the theory of planned behavior. J Dent Hyg Sci 14: 554-562, 2014. https://doi.org/10.17135/jdhs.2014.14.4.554

13. Research Society on Preventative Dentistry: Preventive dentistry: brushing method. Koonja Publising, Seoul, pp.131174, 2007.

14. Choi JI: Oral health education. J Korean Dent Assoc 33: 559-569, 1995.

15. American Dental Hygienists Association: Resources. Retrieved March 13, 2018, from https://www.adha.org/ resources-docs/7221_Proper_Brushing.pdf.

16. NHS Choices: Live well. Retrieved March 13, 2018, from https://www.nhs.uk/Livewell/dentalhealth/Pages/Teethcleani ngguide.aspx.

17. Poyato-Ferrera M, Segura-Egea JJ, Bullón-Fernández P: Comparison of modified Bass technique with normal toothbrushing practices for efficacy in supragingival plaque removal. Int J Dent Hyg 1: 110-114, 2003. https://doi.org/10.1034/j.1601-5037.2003.00018.x

18. Saghazadeh M, Ashayeri N: The comparison between the effectiveness of six different tooth brushing methods on removing dental bacterial plaque. J Dent Med 2: 26-38, 2004.

19. Harnacke D, Mitter S, Lehner M, Munzert J, Deinzer R: 
Improving oral hygiene skills by computer-based training: a randomized controlled comparison of the modified Bass and the Fones techniques. PLoS One 7: e37072, 2012. https://doi.org/10.1371/journal.pone.0037072

20. Ma DS, Park DY, Bae SM, et al.: Preventive technique for periodic professional oral health care. Booksaem Publishing, Seoul, pp.17-30, 2014.

21. Kim CH, Kim GM, Lee JY, Kwon HK, Kim BI: A comparison of tooth brushing methods recommended in different countries. J Korean Acad Oral Health 39: 195-200, 2015. https://doi.org/10.11149/jkaoh.2015.39.3.195

22. Chang KW, Kim JB: An experimental study on the effects of the toothbrushing instructional methods. J Korean Acad Oral Health 11: 85-98, 1987.

23. Shin SJ, Ryu DY, Bae SM, Choi YK: Evaluation on the oral health promotion program effect from some part of community child center. J Dent Hyg Sci 11: 163-171, 2011.

24. Jaime RA, Carvalho TS, Bonini GC, Imparato JCP, Mendes FM: Oral health education program on dental caries incidence for school children. J Clin Pediatr Dent 39: 277-283, 2015. https://doi.org/10.17796/1053-4628-39.3.277

25. Ahn SY, Hwang YS, Han SJ: Effect of oral healthcare program of exemplary kindergartens for prevent the dental caries in Incheon City, Korea. J Dent Hyg Sci 13: 501-509, 2013.

26. Colaizzi LR, Tomar SL, Urdegar SM, Kass SH: Does the structure of dental hygiene instruction impact plaque control in primary school students? J Dent Hyg 89: 180-189, 2015.

27. Eom MR, Jeong DB, Park DY: Enhancement of plaque control score following individualized repeated instruction. J Korean Acad Oral Health 33: 10-18, 2009.

28. Choi YK, Lee MS, Bae SM, Son JH, Ryu DY: Evaluation on the oral health promotion program effect from some part of community child center. J Korea Acad Indust Coop Soc 14: 3850-3857, 2013. https://doi.org/10.5762/KAIS.2013.14.8.3850

29. Wainwrigh J, Sheiham A: An analysis of methods of toothbrushing recommended by dental associations, toothpaste and toothbrush companies and in dental texts. Br Dent J 217: E5, 2014. https://doi.org/10.1038/sj.bdj.2014.651

30. dos Santos AP, Nadanovsky P, de Oliveira BH: Inconsistencies in recommendations on oral hygiene practices for children by professional dental and paediatric organisations in ten countries. Int J Paediatr Dent 21: 223-231, 2011. https://doi.org/10.1111/j.1365-263X.2011.01115.x 